\title{
EVALUACIÓN DE LA SATISFACCIÓN LABORAL DE PROFESORES DE EDUCACIÓN FÍSICA
}

\author{
Raquel Krapp do Nascimento ${ }^{1}$, Gelcemar Oliveira Farias ${ }^{1}$, Marcos Paulo Vaz de Campos \\ Pereira ${ }^{1}$, Jorge Both ${ }^{2}$, Alexandra Folle ${ }^{1}$ \\ quelkrapp@gmail.com; fariasgel@hotmail.com; marcosp.pereira46@gmail.com; \\ jorgeboth@hotmail.com; alexandra.folle@udesc.br \\ ${ }^{1}$ Universidad del Estado de Santa Catarina ${ }^{2}$ Universidad Estadual de Londrina \\ Envío original: 2018-10-22. Reenviado: 2019-04-04. Aceptado: 2019-05-29. \\ Publicado en la versión en español: 2019-10-29
}

Doi: https://doi.org/10.15517/pensarmov.v17i2.39459

\begin{abstract}
RESUMEN
El objetivo del presente estudio fue analizar la satisfacción laboral, considerando las características personales (género, edad y estado civil) de 73 profesores de Educación Física vinculados a la Secretaría Municipal de Educación de la Ciudad de São José (SC). En la recolección de datos, se utilizó un cuestionario sociodemográfico y la escala de evaluación de la calidad de vida laboral percibida por profesores de educación física. Los datos se analizaron por medio de la prueba de U Mann-Whitney. Los resultados revelaron una diferencia significativa de la evaluación general de la satisfacción laboral solo con el grupo de edad (31 años o más> hasta 30 años) de los profesores. Las condiciones de trabajo se diferenciaron en género (mujeres> hombres) y en grupo de edad (31 años o más> hasta 30 años), mientras que la progresión en la carrera, la autonomía en el trabajo y la relevancia social se diferenciaron en la edad (31 años o más de 30 años) de los docentes. Se concluyó que, en comparación con los hombres, las mujeres estaban más satisfechas con las condiciones de trabajo, mientras que los docentes más jóvenes estaban más insatisfechos tanto en la evaluación global como en la mayoría de las dimensiones con respecto a los profesores mayores.
\end{abstract}

Palabras clave: satisfacción laboral, docentes, educación primaria y secundaria.

La enseñanza es una de las profesiones más antiguas y, durante mucho tiempo, fue una de las profesiones más respetadas en el corazón de la sociedad, cuando el maestro era percibido como el fundamento de la escuela y tenía un papel importante en la progresión de los objetivos de las instituciones educativas (Machado et al., 2011; Barros y Pisciotta, 2012). 
Los maestros abarcan una clase desafiada a actualizarse constantemente, a comprender la diversidad de los estudiantes y a diversificar las estrategias de enseñanza para que tengan más oportunidades de cumplir su papel en el proceso de enseñanza y aprendizaje (Araújo, Miranda y Pereira, 2017).

Los cambios en la sociedad han llevado a estos profesionales de la educación a adaptarse ellos mismos y adaptar sus prácticas pedagógicas, con el fin de satisfacer las nuevas demandas de la llamada sociedad moderna, globalizada y una perspectiva diferenciada de otros siglos (Barros y Pisciotta, 2012). En el escenario de las transformaciones sociales, la búsqueda de la mejora de la calidad en la educación es evidente y, en consecuencia, se destaca una mirada dirigida a la satisfacción laboral de la clase docente, la cual desempeña un papel decisivo en la tarea de educar, ya que el nivel de satisfacción laboral se ha relacionado constantemente con el nivel de desempeño de los docentes y su efectividad en el logro de los objetivos educativos (Ramos Holanda et al., 2016). De esa manera, la satisfacción laboral se convierte en un tema extremadamente relevante y complejo al tratarse del desempeño profesional en cualquier área, inclusive en el area de la educación (Araújo et al.,2017).

Al tener en cuenta que la satisfacción laboral interfiere con el desempeño del individuo en su actividad ocupacional (Araújo et al., 2017), se entiende que los niveles más altos de satisfacción laboral, presentados por los docentes, pueden producir mejores resultados escolares, obtenidos por los estudiantes, lo que es un aspecto fundamental en el contexto de la enseñanza (Ramos Holanda et al., 2016), pues el desempeño docente tiene un impacto directo en el aprendizaje y el éxito de los estudiantes (Machado et al., 2011). Sin embargo, la enseñanza parece perder gradualmente su referencia en la esfera social, lo que desencadena cierto demérito de esta profesión y desalienta a los docentes en el ejercicio del magisterio (Barros y Pisciotta, 2012).

La satisfacción laboral se caracteriza como una variable de naturaleza emocional o afectiva, que surge de la evaluación de las condiciones psicosociales del trabajo y la actitud consecuente del trabajador hacia dichas condiciones (Andrade, Barbosa, Souza y Moreira, 2015), lo que representa "[. ..] la suma de los diferentes sentimientos que el trabajador expresa respecto a su actividad laboral "(Oliveira, Ribeiro y Afonso, 2018, p. 83).

En cuanto a la satisfacción laboral docente, una profesión comprendida como sustrato relacional o emocional, es importante comprender cómo se establece la relación positiva o negativa del docente con la profesión y cómo se caracterizan los sentimientos comunicados por los profesores en relación con los diferentes aspectos conciernientes al ejercicio de la docencia y al trabajo pedagógico (Figueiredo y Rojo, 2008; Oliveira et al., 2018). 
En el escenario investigativo de la satisfacción laboral de profesores de la Educación Básica, estudios brasileños (Both et al., 2016; Nascimento, Folle, Rosa y Both, 2016; Ramos Holanda et al., 2016; Veiga, Afonso, Farias, Sinott y Ribeiro , 2017; Oliveira et al., 2018) e internacionales (Chen, 2010; Zhang, 2007; Bota, 2013; Anaya Nieto y López-Martin, 2015) han demostrado, en su mayoría, que los docentes, a pesar de en general estar satisfechos con su profesión y con las relaciones establecidas en el lócus de la intervención profesional, presentan insatisfacción, mayormente con la remuneración que reciben y/o con las condiciones laborales en las escuelas en dónde enseñan. Se enfatiza que estos factores pueden perjudicarles la salud y llevarlos a una calidad de vida más baja, al abandono de la profesión e incluso a enfermedades derivadas del entorno profesional, como el estrés y el síndrome de Burnout (Llorent y Ruiz-Calzado, 2016).

En el caso específico de los profesores de Educación Física, se observa en la producción científica que la satisfacción laboral está asociada con características profesionales, como el vínculo laboral (Folle, Borges, Borges, Coqueiro y Nascimento, 2008a; Nascimento et al., 2016; Veiga et al. al., 2017), multiempleo (Both et al., 2016) y ciclos de desarrollo profesional (Folle, Borges, Coqueiro y Nascimento, 2008b; Both, Borgatto, Lemos, Ciampolini y Nascimento, 2013; Farias, Both, Folle, Pinto y Nascimento, 2015; Rocha, Squarcini, Cardoso y Farias, 2016; Veiga et al., 2017). Por otro lado, las variables relacionadas con las características personales (género, edad y estado civil) de esta clase trabajadora son poco investigadas (Folle et al., 2008a, 2008b; Moreira, Nascimento, Sonoo, Both, 2010; Both, Nascimento, Sonoo, Lemos y Borgatto, 2014, MuñozMendez, Gómez-Mármol y Martínez, 2017). Estos estudios no encontraron diferencias significativas entre hombres y mujeres, además, se evidenciaron resultados no concluyentes con respecto al grupo de edad (los maestros más jóvenes a veces están más satisfechos y otras veces más insatisfechos) e identificaron que los docentes casados tienen una mayor percepción de satisfacción laboral que los solteros.

Por lo tanto, al no haber congruencia en los resultados presentados en los documentos con respecto a ciertos factores individuales asociados con la satisfacción laboral y con el fin de ampliar las discusiones sobre este tema en el contexto de la enseñanza educativa brasileña, esta investigación tiene como objetivo analizar la satisfacción laboral de los profesores de Educación Física de la red municipal de educación de São José (Santa Catarina, Brasil), considerando el género, grupo de edad y el estado civil.

\section{METODOLOGÍA}

Participantes 
La encuesta se realizó en 2016 en São José, una ciudad costera en el sur de Brasil, con una población estimada de 242,927 habitantes y un índice de desarrollo humano de 0.809. La Secretaría Municipal de Educación cuenta con 36 centros de educación infantil y 23 centros educativos municipales, que atienden a aproximadamente 23.890 estudiantes. La población investigada abarcó los 95 docentes que enseñaban el componente curricular de Educación Física en esta red educativa.

La muestra no probabilística (todos fueron invitados a participar) estaba conformada por 73 docentes, con edad promedio de 36,8 ( $\pm 10,1)$ años, que correspondían al $74,8 \%$ de la población. Entre los participantes del estudio: $56,2 \%$ eran mujeres y $43,8 \%$ hombres; el $58,9 \%$ tenía pareja y el $41,1 \%$ no tenía pareja; el $37,0 \%$ de los maestros tenían hasta 30 años y el $63,0 \%$ tenían 31 años o más. Además, se destaca que el $53,5 \%$ de los profesores tenía hasta nueve años de experiencia docente y el $46,5 \%$ tenía 10 años o más.

Instrumentos de medición

En la recolección de datos se utilizaron los siguientes instrumentos autoaplicados:

a) Cuestionario sociodemográfico, preparado por los autores, que consta de dos partes, a saber: información personal (género, edad y estado civil); e información profesional (formación académica, años completos de docencia, tiempo de servicio en la red educativa, vínculo laboral, horas trabajadas en general, horas trabajadas en la red educativa, lugar de trabajo, etapa de la enseñanza, docencia como principal fuente de ingresos, otras fuentes de ingresos, número de escuelas en que actúan, número de clases impartidas). En este estudio, se utilizaron las variables relacionadas con la información personal.

b) Escala de evaluación de la calidad de vida laboral percibida por los profesores de educación física de primaria y secundaria - CVL-PEF (Both et al., 2006), que evaluó la percepción de los profesores de educación física con respecto a la satisfacción laboral.

La escala CVL-PEF se compone de 34 preguntas que se distribuyen en ocho dimensiones: remuneración y compensación; condiciones de trabajo; autonomía en el trabajo; progresión profesional; integración social; leyes y normas laborales; trabajo y equilibrio entre la vida laboral y personal; relevancia social del trabajo; y con posibilidades de respuesta que van desde 1 (totalmente en desacuerdo) a 7 (totalmente de acuerdo). En la validación del contenido, se presentaron tasas superiores al $70 \%$ de concordancia entre los expertos y en la evaluación de la reproducibilidad, el $94,1 \%$ de las preguntas obtuvieron un fuerte coeficiente de correlación de Spearman $(>0,60)$. La consistencia interna general del instrumento, verificada por medio del Alfa de Cronbach, se consideró excelente $(0,94)$ (Both et al., 2006).

Procedimientos 
Para llevar a cabo la investigación, se contactó al coordinador de Educación Física de la Secretaría de Educación y se programó una reunión para presentar el estudio y obtener el consentimiento para su realización. El estudio fue aprobado por el Comité de ética en investigación con seres humanos de una universidad pública del sur de Brasil (dictamen 974.054/2015). La participación de los docentes se llevó a cabo mediante la firma del Término de consentimiento informado.

Los datos se recolectaron en dos reuniones de formación continua ofrecidas por la Secretaría Municipal de Educación, impartida una vez al mes y es parte de las actividades profesionales de los profesores de la red de Educación Física. Las reuniones se llevaron a cabo en la Casa do Educador y la Secretaría de Educación Municipal de São José. Los cuestionarios se entregaron a los docentes en el intervalo de las actividades de formación continua y recogidos al finalizar su llenado.

\section{Tratamiento estadístico}

Inicialmente, los profesores se clasificaron según el género (femenino y masculino), considerando sus respuestas con respecto a la edad (hasta 30 años: edad adulta temprana y 31 años o más: proceso de consolidación familiar y profesional), agrupándolos según la división de dos ciclos vitales, propuesta por Sikes (1985), que presentó similitud con la realidad del sur de Brasil (Both et al., 2014), y con respecto al estado civil (con pareja y sin pareja), considerando con la pareja a los maestros que respondieron las opciones de casado o unión estable, y sin pareja los maestros que indicaron las opciones de soltero, divorciado o viudo.

Concerniente a la satisfacción laboral, se utilizaron las ecuaciones de ponderación de Lemos (2007) para transformar y clasificar los puntajes $(-100.00$ a +100.00$)$, utilizando la prueba de Kolmogorov-Smirnov para verificar la normalidad de los datos. Considerando la anormal distribución de los datos, se utilizó la mediana (Md) como la medida de tendencia central y el rango intercuartil (Q1:Q3) como medida de dispersión. En el análisis estadístico inferencial, se utilizó la prueba $U$ Mann-Whitney para comparar la satisfacción laboral con las variables sociodemográficas. Todos los análisis estadísticos se realizaron con la ayuda del software SPSS, versión 20.0. En todos los análisis estadísticos, se destacó el nivel de confianza adoptado en el estudio, que fue del 95,0\% $(p<0,05)$.

\section{RESULTADOS}

El análisis de la satisfacción laboral según el género de los profesores de Educación Física (Tabla 1) mostró una diferencia significativa solo con la dimensión de condiciones de 
trabajo $(p=0.01)$, mostrando que las mujeres estaban más satisfechas que los hombres.

Tabla 1.

Satisfacción laboral de profesores de Educación Física, según el género.

\begin{tabular}{|c|c|c|c|}
\hline \multirow[b]{2}{*}{ Dimensiones del trabajo } & \multicolumn{2}{|c|}{ Género } & \multirow[b]{2}{*}{$\mathbf{p}$} \\
\hline & $\begin{array}{l}\text { Femenino } \\
\text { Md }(Q 1 ; Q 3)\end{array}$ & $\begin{array}{l}\text { Masculino } \\
\text { Md }(Q 1 ; Q 3)\end{array}$ & \\
\hline Evaluación general & $75,00(50,00 ; 87,50)$ & $62,50(3,13 ; 75,00)$ & 0,06 \\
\hline Remuneración & $8,33(-25,00 ; 33,33)$ & $-4,17(-43,75 ; 16,67)$ & 0,17 \\
\hline Condiciones laborales & $33,33(0,00 ; 50,00)$ & $2,78(-31,94 ; 26,39)$ & 0,01 \\
\hline Autonomía en el trabajo & $66,67(22,22 ; 77,78)$ & $50,00(22,22 ; 76,39)$ & 0,60 \\
\hline Progresión profesional & $50,00(25,00 ; 75,00)$ & $45,84(16,67 ; 58,33)$ & 0,26 \\
\hline Integración social en el trabajo & $33,33(13,33 ; 50,00)$ & $23,34(-5,00 ; 40,00)$ & 0,14 \\
\hline Leys y regulaciones laborales & $50,00(29-17 ; 75,00)$ & $50,00(10,42 ; 72,92)$ & 0,28 \\
\hline $\begin{array}{l}\text { Trabajo y equilibrio entre la vida } \\
\text { laboral y personal }\end{array}$ & $16,67(-16,67 ; 58,34)$ & $8,34(-29,17 ; 50,00)$ & 0,60 \\
\hline Relevancia social en el trabajo & $77,78(44,44 ; 88,89)$ & $55,56(36,11 ; 77,78)$ & 0,08 \\
\hline
\end{tabular}

Etiqueta: Md - mediana (medición de la tendencia central); Q1:Q3 - rango intercuartil (medición de dispersión); $\mathrm{p}$ - probabilidad estimada por la Prueba U de Mann Whitney.

Al evaluar la satisfacción laboral de los profesores según la edad (Tabla 2), se encontró una diferencia estadísticamente significativa en la evaluación general $(p=0.05)$ y en las dimensiones de condiciones de trabajo $(p=0.02)$, de autonomía en $(p<0.01)$, de progresión profesional $(p<0.01)$ y de relevancia social del trabajo $(p<0.01)$, con docentes de hasta 30 años menos satisfechos que docentes con más de 31 años

Tabla 2.

Satisfacción laboral de los profesores de Educación Física, según el grupo de edad.

\begin{tabular}{|c|c|c|c|}
\hline \multirow[b]{2}{*}{ Satisfacción Laboral } & \multicolumn{2}{|c|}{ Grupo de Edad } & \\
\hline & $\begin{array}{l}\text { Hasta } 30 \text { Años } \\
\text { Md (Q1;Q3) }\end{array}$ & $\begin{array}{l}\text { 31 o más años } \\
\text { Md }(Q 1 ; Q 3)\end{array}$ & \\
\hline Evaluación global & $62,50(0,00 ; 75,00)$ & $66,97(38,40 ; 87,50)$ & 0,05 \\
\hline Remuneración & $0,00(-16,67 ; 25,00)$ & $0,00(-43,75 ; 33,33)$ & 0,78 \\
\hline Condiciones laborales & $-5,56(-22,22 ; 27,78)$ & $30,56(0,00 ; 51,39)$ & 0,02 \\
\hline Autonomía en el trabajo & $44,44(16,67 ; 61,11)$ & $66,67(38,89 ; 83,33)$ & $<0,01$ \\
\hline Progresión profesional & $25,00(0,00 ; 41,67)$ & $58,33(31,25 ; 75,00)$ & $<0,01$ \\
\hline Integración social en el trabajo & $26,67(-26,67 ; 40,00)$ & $33,33(13,33 ; 60,00)$ & 0,07 \\
\hline Leys y regulaciones laborales & $50,00(8,33 ; 66,67)$ & $54,17(25,00 ; 75,00)$ & 0,12 \\
\hline $\begin{array}{l}\text { Trabajo y equilibrio entre la vida } \\
\text { laboral y personal }\end{array}$ & 0) & 17) & 0,59 \\
\hline Relevancia social en el trabajo & $44,44(22,22 ; 77,78)$ & $77,78(52,78 ; 88,89)$ & $<0,01$ \\
\hline
\end{tabular}

Etiqueta: Md - mediana (medición de la tendencia central); Q1:Q3 - rango intercuartil (medición de dispersión); $\mathrm{p}$ - probabilidad estimada por la Prueba U de Mann Whitney.

Al analizar la satisfacción laboral, considerando el estado civil de los profesores de Educación Física de la red municipal de educación (Tabla 3), no hubo diferencias estadísticamente significativas en la evaluación general ni en las dimensiones de trabajo evaluado entre docentes sin y con pareja. 
Tabla 3.

Satisfacción laboral de los profesores de Educación Física, según el estado civil.

\begin{tabular}{|c|c|c|c|}
\hline \multirow[b]{2}{*}{ Satisfacción Laboral } & \multicolumn{2}{|c|}{ Estado Civil } & \multirow[b]{2}{*}{$\mathbf{p}$} \\
\hline & $\begin{array}{l}\text { Sin Pareja } \\
\text { Md (Q1;Q3) }\end{array}$ & $\begin{array}{l}\text { Con Pareja } \\
\text { Md (Q1;Q3) }\end{array}$ & \\
\hline Evaluación global & $62,50(42,86 ; 87,50)$ & $56,25(9,38 ; 75,00)$ & 0,21 \\
\hline Remuneración & $0,00(-16,67 ; 25,00)$ & $0,00(-52,08 ; 27,08)$ & 0,72 \\
\hline Condiciones laborales & $22,22(-11,11 ; 38,89)$ & $13,89(-25,00 ; 50,00)$ & 0,70 \\
\hline Autonomía en el trabajo & $66,67(22,22 ; 77,78)$ & $55,56(20,83 ; 72,22)$ & 0,23 \\
\hline Progresión profesional & $50,00(25,00 ; 66,67)$ & $37,50(12,50 ; 68,75)$ & 0,55 \\
\hline Integración social en el trabajo & $33,33(13,33 ; 60,00)$ & $26,67(-15,00 ; 40,00)$ & 0,06 \\
\hline Leyes y regulaciones laborales & $58,33(25,00 ; 75,00)$ & $50,00(22,92 ; 60,42)$ & 0,15 \\
\hline $\begin{array}{l}\text { Trabajo y equilibrio entre la vida } \\
\text { laboral y personal }\end{array}$ & $3(1667$. & 0) & 0,33 \\
\hline Relevancia social en el trabajo & $66,67(44,44 ; 88,89)$ & $55,56(33,33 ; 77,78)$ & 0,20 \\
\hline
\end{tabular}

Etiqueta: Md - mediana (medición de la tendencia central); Q1:Q3 - rango intercuartil (medición de dispersión); $\mathrm{p}$ - probabilidad estimada por la Prueba U de Mann Whitney.

\section{DISCUSIÓN}

El objetivo de este estudio fue analizar la satisfacción laboral de los profesores de Educación Física, según el género, la edad y el estado civil. El análisis de la evaluación general de la satisfacción laboral de acuerdo al género de los profesores de educación física no reveló diferencias relevantes, aunque las mujeres tuvieron tasas más altas de satisfacción.

Estudios realizados con profesores del Reino Unido (Crossman y Harris, 2006), de Brasil (Folle et al., 2008a, 2008b; Araújo et al., 2017), de Colombia (Rojas, Zapata y Grisales, 2009), de Turquía (Demirtas, 2010), Sudáfrica (Strydom, Nortjé, Beukes, Esterhuyse y Westhuizen, 2012) y España (Muñoz-Méndez et al., 2017) tampoco encontraron diferencias significativas de género en la evaluación global de satisfacción

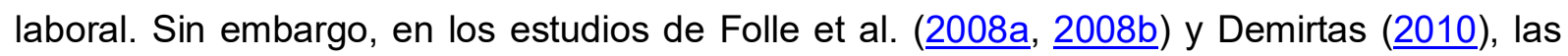
mujeres estaban más satisfechas en el ambiente laboral que los hombres. Por otro lado, en las investigaciones de Crossman y Harris (2006) y de Both, Borgatto, Lemos, Ciampolini y Nascimento (2017), los hombres estaban más satisfechos que las mujeres.

La investigación realizada con profesores israelíes ha encontrado una diferencia estadísticamente significativa entre los géneros, lo que demuestra que las profesoras tienen altos niveles de satisfacción, mientras que sus pares tienen bajos niveles de satisfacción en 
la enseñanza escolar (Bogler, 2002). En este contexto, Machado y Silva (2014) corroboran que, específicamente en términos de encuestas sobre satisfacción laboral y género de los empleados, las mujeres han mostrado niveles de satisfacción superiores que los hombres. La satisfacción laboral al establecer una relación con las creencias y las expectativas en el desempeño docente, realmente se centra en la percepción de que las profesoras se muestran más optimistas sobre su profesión en detrimento de los hombres (Farias, Nascimento, Graça y Batista, 2011).

Con respecto a la comparación de las dimensiones del trabajo con el género de los profesores de Educación Física, se comprobó que las mujeres estaban más satisfechas con las condiciones laborales. Se divulgaron resultados similares en una investigación con profesores griegos (Koustelios, 2001), que evidenciaron el género como un predictor significativo de las condiciones laborales, en el que las mujeres están más satisfechas. Por otro lado, los resultados presentados en un estudio con profesores de Educación Física de escuelas públicas en el sur de Brasil revelaron que los hombres están significativamente más satisfechos con las condiciones laborales y con el trabajo y también con el equilibrio entre la vida laboral y personal (Both et al., 2017).

Del mismo modo, la investigación con profesores de países africanos mostró que los hombres estaban más satisfechos con algunos componentes de satisfacción laboral referentes a la estructura y los procesos organizacionales, ya que estos consideran que el sistema de promoción, recompensas e incentivos es más justo, además de que las escuelas permiten a los hombres mayor autonomía para usar sus habilidades (Al-Mashaan, 2003). Se hace evidente en las relaciones establecidas en el ámbito escolar que docentes varones, de acuerdo con la demanda de actividades que serán desempeñadas, tienden a presentar una mayor percepción de satisfacción en vista de la postura y la ocupación de los puestos de gestión. Aunque históricamente, la carrera de la profesión docente tiene un sesgo de feminización (Valle, 2003).

En lo que se refiere a la satisfacción laboral y el grupo de edad de los profesores de Educación Física, se evidenció que con el aumento de la edad, los profesores están más satisfechos con el contexto de su intervención profesional. Se presume que los profesionales de más edad, y probablemente con más horas de trabajo, tienden a adaptarse al trabajo y a concebir una perspectiva más objetiva, en el sentido de atribuir valores más altos a las facetas del trabajo, las cuales, los trabajadores más jóvenes, a veces, las consideran insatisfactorias (Carrillo-García, Solano-Ruíz, Martínez-Roche y Gómez-García, 2013). No obstante, Crossman y Harris (2006) informaron resultados divergentes, en los que los profesores más jóvenes del Reino Unido (hasta 30 años) tuvieron una mayor satisfacción.

Al comparar las dimensiones de satisfacción laboral y edad, los profesores 
investigados en este estudio, que tenían hasta 30 años, estaban más satisfechos con las dimensiones de clases de trabajo, autonomía laboral, progresión profesional y de relevancia social del trabajo. Los estudios realizados con profesores de educación física, que trabajan en redes públicas en la región sur de Brasil, también encontraron resultados divergentes con respecto a la evaluación de las dimensiones del trabajo. En el caso brasileño, Both et al. (2014) encontraron que los profesores de educación física de 30 a 39 años estaban más insatisfechos con las condiciones laborales. A su vez, Moreira et al. (2010) mostraron que con el aumento de la edad, los profesores estaban más satisfechos con las condiciones laborales. Además, descubrieron que los profesores estaban satisfechos con la autonomía en el trabajo, el progreso profesional, las leyes y normas laborales y la relevancia social, aunque los valores de satisfacción disminuen con la edad avanzada.

Al comparar el estado civil con la satisfacción laboral de los profesores de Educación Física, las diferencias no fueron estadísticamente significativas, a pesar de observar que los profesores sin pareja presentaron tasas de satisfacción superiores. En una investigación con docentes griegos (Koustelios, 2001), el estado civil tampoco se caracterizó como un predictor de la satisfacción laboral. Por el contrario, Mwamwenda (1997) y Peterson y Dunnagan (1998) identificaron niveles más altos de satisfacción laboral para el grupo de profesores casados de las escuelas Transkei en Sudáfrica y las universidades de EE. UU., respectivamente. Por otro lado, aunque no haya encontrado diferencias estadísticamente significativas entre la satisfacción laboral y el estado civil, Furtado (2015) reveló que los profesores portugueses con pareja tenían niveles más altos de satisfacción laboral.

La información presentada sobre la satisfacción laboral y el estado civil de los profesores corrobora la indicación de Azim, Haque y Chowdhury (2013) de que no existe una conclusión sobre el efecto del estado civil sobre la satisfacción laboral. Sin embargo, hubo una tendencia de mayor satisfacción laboral en los empleados casados. Una explicación para este hallazgo se relaciona con el hecho de que el matrimonio impone grandes responsabilidades que conlleva a la búsqueda de un trabajo más estimado y relevante, así como la satisfacción laboral se caracteriza por ser necesaria para lograr dicha estabilidad, lo que los orienta a esforzarse por ajustar los aspectos que no les satisfacen.

Se concluye que la evaluación general de la satisfacción laboral es diferente según el grupo de edad de los profesores de Educación Física (profesores mayores y más satisfechos). Sin embargo, al considerar el género y el estado civil de los profesores, no hay diferencia en la evaluación general de la satisfacción laboral. Las condiciones de trabajo diferían en comparación con el género (mujeres más satisfechas) y el grupo de edad (profesores mayores más satisfechos), mientras que la progresión profesional, la autonomía en el trabajo y la relevancia social diferían en relación con la edad de los profesores (profesores mayores más satisfechos con estas dimensiones). 
La limitación de esta investigación se refiere al hecho de que se realizó con profesores de una única red escolar. Sin embargo, en la realidad brasileña hay pocas investigaciones que intentaron evaluar características detalladas de las redes municipales de educación. Se recomienda que los nuevos estudios investiguen la realidad de otras ciudades para cubrir una mayor parte de los municipios y, en consecuencia, identificacar las diferentes carreras docentes determinadas por las ordenanzas municipales de los magisterios.

\section{REFERENCIAS}

Al-Mashaan, O.S. (2003). Comparison between kuwaiti and egyptian teachers in type a behavior and job satisfaction: a cross-cultural study. Social Behavior and Personality, 31(5), 523-534. doi: https://doi.org/10.2224/sbp.2003.31.5.523

Anaya Nieto, D. y López-Martin, E. (2015). Job satisfaction of secondary school teachers. Revista de Investigación Educativa, 33(2), 435-452. doi: https://doi.org/10.6018/rie.33.2.202841

Andrade, T.F., Barbosa, S.C., Souza, S. y Moreira, J.S. (2015). Valores humanos e satisfação no trabalho de professores e servidores técnico-administrativos de uma universidade pública. Revista de Psicologia: Organizações e Trabalho, 15(4), 397406. doi: https://doi.org/10.17652/rpot/2015.4.486

Araújo, T.S., Miranda, G.J. y Pereira, J.M. (2017). Satisfação dos professores de Contabilidade no Brasil. Revista Contabilidade e Finanças, 28(74), 264-281. doi: https://doi.org/10.1590/1808-057x201703420

Azim, M.T., Haque, M.M. y Chowdhury, R.A. (2013). Gender, marital status and job satisfaction an empirical study. International Review of Management and Business Research, 2(2), 488-498. Recuperado de http://citeseerx.ist.psu.edu/viewdoc/summary?doi=10.1.1.682.2246

Barros, S.S.P. y Pisciotta, R.M. (2012). Profissão docente: o conceito da docência no âmbito social. Revista Interfaces, 4(2), 45-47. Recuperado de http://uniesp.edu.br/sites/_biblioteca/revistas/20170419174650.pdf

Bogler, R. (2002). Two profiles of schoolteachers: a discriminant analysis of job satisfaction. Teaching and Teacher Education, 18(6), 665-673. doi: https://doi.org/10.1016/S0742051X(02)00026-4

Bota, O.A. (2013). Job satisfaction of teachers. Procedia - Social and Behavioral Sciences, 83, 634-638. doi: https://doi.org/10.1016/j.sbspro.2013.06.120

Both, J., Borgatto, A.F., Lemos, C.A.F., Ciampolini, V. y Nascimento, J.V. (2017). Physical Education teachers wellbeing and its relation with gender. Motricidade, 13(2), 23-32. doi: https://doi.org/10.6063/motricidade.10165

Both, J., do Nascimento, J.V., Fogliarini Lemos, C.A., Donegá, A.L., Kodama Pertille Ramos, M.H., Petroski, E.C. y da Silva Duarte, M.F. (2006). Qualidade de vida no trabalho percebida por professores de Educação Física. Revista Brasileira de 
Cineantropometria e Desempenho Humano, 8(2),45-52. Recuperado de https://periodicos.ufsc.br/index.php/rbcdh/article/view/3884/16669

Both, J., Nascimento, J.V., Sonoo, C.N., Lemos, C.A.F. y Borgatto, A.F. (2013). Bem estar do trabalhador docente em Educação Física ao longo da carreira. Revista de Educação Física/UEM, 24(2), 233-246. doi: https://doi.org/10.4025/reveducfis.v24i2.16114

Both, J., Nascimento, J.V., Sonoo, K.N., Lemos, C.A.F. y Borgatto, A.F. (2014). Bem estar do trabalhador docente de Educação Física da região sul do Brasil de acordo com os ciclos vitais. Revista Brasileira de Educação Física e Esporte, 28(1), 77-93. doi: http://dx.doi.org/10.1590/S1807-55092014000100077

Both, J., Ferreti Borgatto, A., Noriko Sonoo, C., Fogliarini Lemos, C.A., Ciampolini, V. y Vieira do Nscimento, J. (2016). Multiple job holding associated with the wellbeing of physical education teachers in southern Brazil. Educacion Fisica y Deporte, 35(1), 110. doi: http://doi.org/10.17533/udea.efyd.v35n1a05

Carrillo-García, C., Solano-Ruíz, M.C., Martínez-Roche, M.E. y Gómez-García, C.I. (2013). Influência do gênero e da idade: satisfação no trabalho de profissionais da saúde. Revista Latino-Americana de Enfermagem, 21(6), 1314-1320. doi: https://doi.org/10.1590/0104-1169.3224.2369

Chen, J.J. (2010). Chinese middle school teacher job satisfaction and its relationships with teacher moving. Asia Pacific Education Review, 11(3), 263-272. doi: https://doi.org/10.1007/s12564-010-9085-1

Crossman, A. y Harris, P. (2006). Job satisfaction of secondary school teachers. Educational Management Administration y Leadership, 34(1), 29-46. doi: https://doi.org/10.5539/ass.v7n8p203

Demirtas, Z. (2010). Teachers' job satisfaction levels. Procedia - Social and Behavioral Sciences, 9, 1069-1073. doi: https://doi.org/10.1016/j.sbspro.2010.12.287

Farias, G.O., Both, J., Folle, A., Pinto, M.G. y Nascimento, J.V. (2015). Satisfação no trabalho de professores de Educação Física do magistério público municipal de Porto Alegre. Revista Brasileira de Ciência e Movimento, 23(3), 5-13. doi: http://dx.doi.org/10.18511/0103-1716/rbcm.v23n3p5-13

Farias, G.O., Nascimento, J.V., Graça, A. y Batista, P.M.F. (2011). Crenças e expectativas constituídas ao longo da carreira docente em Educação Física. Revista de Educação Física, 22(4), 497-509. doi: https://doi.org/10.4025/reveducfis.v22i4.10651

Figueiredo, M. y Rojo, V.A. (2008). Satisfacción con la profesión de profesor de Enseñanza Secundaria en Portugal. Revista Española de Orientación y Psicopedagogía, 19(3), 255-269. doi: https://doi.org/10.5944/reop.vol.19.num.3.2008.11427

Folle, A., Borges, L.J., Coqueiro, R.S. y Nascimento, J.V. (2008a). Nível de (in)satisfação de professores de Educação Física com o trabalho realizado nos Centros de Educação Infantil no estado de Santa Catarina. Revista Brasileira de Ciência e Movimento, 16(4), 1-21. Recuperado de https://portalrevistas.ucb.br/index.php/RBCM/article/view/834

Folle, A., Borges, L.J., Coqueiro, R.S. y Nascimento, J.V. (2008b). Nível de (in)satisfação 
profissional de professores de Educação Física da Educação Infantil. Motriz, 14(2), 124-134. Recuperado de http://www.periodicos.rc.biblioteca.unesp.br/index.php/motriz/article/view/1198/1714

Furtado, S.C.O. (2015). Satisfação profissional e bem-estar subjetivo dos professores em pré-reforma. (Tese de Mestrado). Universidade dos Açores, Departamento de Ciências da Educação, Ponta Delgada, Portugal. Recuperado de https://repositorio.uac.pt/handle/10400.3/3778

Koustelios, A.D. (2001). Personal characteristics and job satisfaction of greek teachers. International Journal of Educational Management, 15(7), 354-358. doi: https://doi.org/10.1108/EUM0000000005931

Lemos, C.A.F. (2007). Qualidade de vida na carreira profissional de professores de Educação Física do magistério público estadual/RS. (Tese de Mestrado). Universidade Federal de Santa Catarina, Centro de Desportos, Florianópolis, Brasil. Recuperado de https://repositorio.ufsc.br/handle/123456789/89948

Llorent, V.J. y Ruiz-Calzado, I. (2016). Burnout and its relation to sociodemographic variables among education professionals working with people with disabilities in Córdoba (Spain). Ciência \& Saúde Coletiva, 21(10), 3287-3295. doi: https://doi.org/10.1590/1413-812320152110.00732015

Machado, D.C. y Silva, A.F. (2014). Um indicador de não satisfação no trabalho e a mobilidade do mercado de trabalho: um estudo para homens e mulheres. Nova Economia, 24(1), 123-140. doi: http://dx.doi.org/10.1590/0103-6351/1410

Machado, M. L., Meira Soares, V., Ferreira Brites, J., Farhangmehr, M. y Gouveia, O. (2011). Uma análise da satisfação e da motivação dos docentes no Ensino Superior português. Revista Lusófona de Educação, 17, 167-181. Recuperado de http://www.scielo.mec.pt/pdf/rle/n17/n17a11.pdf

Moreira, H.R., Nascimento, J.V., Sonoo, C.N. y Both, J. (2010). Qualidade de vida do trabalhador docente e os ciclos vitais de professores de Educação Física do Estado do Paraná, Brasil. Revista Brasileira de Ciência e Movimento, 18(3), 12-20. doi: https://doi.org/10.4025/reveducfis.v22i2.8690

Muñoz-Méndez, T., Gómez-Mármol, A. y Martínez, B.J.S.A. (2017). Job satisfaction among preschool, elementary and secondary school teachers. Revista Gestion de la Educacion, 7(1), 161-177. doi: https://doi.org/10.1108/09578230410534676

Mwamwenda, T.S. (1997). Marital status and teachers' job satisfaction. Psychological Reports, 80(2), 521-522. doi: https://doi.org/10.2466/pr0.1997.80.2.521

Nascimento, R.K., Folle, A., Rosa, A.I. y Both, J. (2016). Job satisfaction among Physical Education teachers from the municipal network of São José-SC. Journal of Physical Education, 27(4), e2740. doi: https://doi.org/10.4025/jphyseduc.v27i1.2740

Oliveira, I.B., Ribeiro, J.A.B. y Afonso, M.R. (2018). Satisfação com a profissão: um estudo com professores de Educação Física. Pensar a Prática, 21(1), 82-95. doi: https://doi.org/10.5216/rpp.v21i1.39403

Peterson, M. y Dunnagan, T. (1998). Analysis of a worksite health promotion program's impact on job satisfaction. Journal of Occupational and Environmental Medicine, 
40(11),

973-979.

Recuperado

de

https://journals.Iww.com/joem/Abstract/1998/11000/Analysis_of_a_Worksite_Health_ Promotion_Program_s.7.aspx

Ramos Holanda, M.F., de Oliveira Fernandez, A.P., Leal Soares Ramos, E.M., da Costa e Silva, S.S. y Ramos Pontes, F.A. (2016). Satisfação no trabalho docente: uma análise a partir do modelo social cognitivo de satisfação no trabalho e da eficácia coletiva docente. Estudos de Psicologia, 21(2), 179-191. doi: http://dx.doi.org/10.5935/1678-4669.20160018.

Rocha, S.V., Squarcini, C.F., Cardoso, J.P. y Farias, G.O. (2016). Características ocupacionais e estilo de vida de professores em um município do nordeste brasileiro. Revista de Salud Publica, 18(2), 214-225. doi: http://dx.doi.org/10.15446/rsap.v18n2.47636

Rojas, M.L., Zapata, J.A. y Grisales H. (2009). Síndrome de burnout y satisfacción laboral en docentes de una institución de educación superior, Medellín, 2008. Revista Facultad Nacional de Salud Pública, 27(2), 198-210. Recuperado de http://www.redalyc.org/pdf/120/12011791011.pdf

Strydom, L., Nortjé, N., Beukes, R., Esterhuyse, K. y Westhuizen, J. (2012). Job satisfaction amongst teachers at special needs schools. South African Journal of Education, 32(30), 255-266. doi: https://doi.org/10.15700/saje.v32n3a582

Sikes P. (1985). The life cycle of the teacher. En: Ball, S.J., Goodson I.F. (Org.). Teachers' lives and careers. London: Falmer.

Valle, I. (2003). A era da profissionalização: formação e socialização profissional do corpo docente de $1^{a}$ a $4^{a}$ série. Florianópolis, Brasil: Cidade Futura.

Veiga, R.F., Afonso, M.A., Frias, G.O., Sinott, E.C. y Ribeiro, J.A.B. (2017). Qualidade de vida no trabalho: contexto de atuação profissional e carreira docente. Pensar a Prática, 20(2), 333-348. doi: https://doi.org/10.5216/rpp.v20i2.36798

Zhang, Z. S. (2007). Study of job satisfaction among elementary school teachers in Shanghai. Chinese Education \& Society, 40(5), 40-46. doi: https://doi.org/10.2753/CED1061-1932400505. 